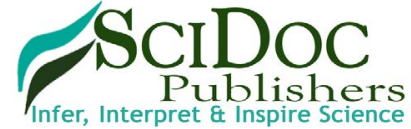

International Journal of Anesthesiology \& Research (IJAR)

ISSN 2332-2780

\title{
"STOP BANG" Questionnaire: Validity to Screen Obstructive Sleep Apnea Syndrome in North African Population
}

Review Article

Kharrat $\mathrm{A}^{1 *}$, Cheikhrouhou $\mathrm{H}^{1}$, Msaad $\mathrm{S}^{2}$, Karoui $\mathrm{A}^{1}$, Kammoun $\mathrm{S}^{2}$

${ }^{1}$ Department of Anesthesiology, Habib Bourguiba Hospital, Sfax, Tunisia.

${ }^{2}$ Department of Pneumology, Hedi Chaker Hospital, Sfax, Tunisia.

\section{Abstract}

Background: The aim of this study was to validate the predictive value of the questionnaire "STOP BANG" in the detection of obstructive sleep apnea syndrome syndrome (OSAS) and to identify patients at high risk of OSASS in anesthesia consultation in North African population.

Methods: Prospective clinical study, consecutive, about 759 patients proposed for elective surgery and enjoying a remote anesthesia consultation in H. Bourguiba hospital in Sfax, Tunisia. Included patients answered the questionnaire "STOP BANG". Patients with a score below 3 are considered low risk and represent the group (1). Patients with a score greater than or equal to 3 are considered high risk and represented the group (2). All patients were investigated by ventilator polygraphy to confirm the diagnosis of obstructive apnea syndrome. The apnea hypopnea index (AHI) greater than or equal to 5 confirmed the diagnosis of OSAS.

Results: One hundred and fifty patients were included in the study. 41 patients had low risk of OSAS and represented the group (1) and $27.33 \%$ of included patients. 109 patients had high risk of OSAS and represented the group (2) and $72.66 \%$ of included patients. The diagnosis of OSAS was confirmed among 127 patients including 99 in the group (1) and 28 in the group (2). In our study results showed that the sensitivity of the score STOP BANG questionnaire was to the order of $87.1 \%$, specificity was $47.8 \%$, with positive and negative predictive values $90 \%$ and 40.74 respectively. The sensitivity of the questionnaire increased significantly with increasing $\mathrm{AHI}$, it was $91.3 \%$ for $\mathrm{IAH} \geq 15 / \mathrm{H}$ and $93.9 \%$ for $\mathrm{AHI} \geq 30 / \mathrm{H}$. Similarly, the predictive negative value of the questionnaire increased parallel with the increase in the AHI, it was $70.37 \%$ for $\mathrm{AHI} \geq 15 / \mathrm{H}$ and $88.9 \%$ for $\mathrm{AHI} \geq 30 / \mathrm{H}$.

Conclusion: The sensitivity, specificity, positive and negative predictive value vary by IAH and score STOP BANG. These results show that the STOP BANG questionnaire is a good simple screening tool, easy, particularly suitable for anesthesia consultation. The questionnaire has excellent sensitivity and specificity to identify patients at high risk of OSAS.

Keywords: STOP BANG; Obstructive Sleep Apnea Syndrome; Validity; Ventilatory Polygraphy; Sensibility; Specificity.

\section{Background}

Obstructive sleep apnea syndrome (OSAS) is a disorder a disorder characterized by repetitive periods of apnea and hypopnea during sleep [1]. It's a common disorder as it affects 3-7\% of adult male and $2-5 \%$ of adult female population [2]. OSAS increases mortality compared with general population [3] and raises the cardiovascular, respiratory, cerebrovascular and metabolic risk [4]. In addition, OSAS exposed to anesthetic complications, such as, difficulty of management of upper airway, difficult mask ventilation and intubation, postoperative obstruction of airway and prolongation of hospital stay [5, 6]. The diagnosis of OSAS remains imperative in patients at risk before general anesthesia. Polysomnography is the gold standard for confirming the diagnosis of OSAS [7]. This test is expensive, time consuming and requires a significant waiting period which may delay surgery. Faced with these constraints, OSAS screening tool is essential to limit exploration and screen patients at risk requiring the use of polysomnography. "STOP BANG" questionnaire, created in 2008 by the American Society of Anesthesiology (ASA), has proven effective for screening high-risk patients to develop OSAS [8]. The predictive performance of the questionnaire clearly

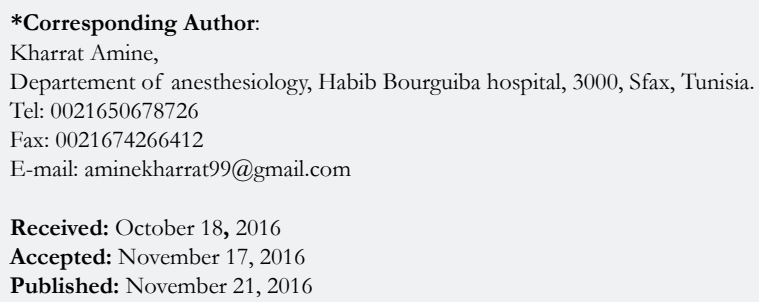

Citation: Kharrat A, Cheikhrouhou H, Msaad S, Karoui A, Kammoun S (2016) "STOP BANG" Questionnaire: Validity to Screen Obstructive Sleep Apnea Syndrome in North African Population. Int J Anesth Res. 4(11), 352-357. doi: http://dx.doi.org/10.19070/2332-2780-1600073

Copyright: Kharrat $\mathbf{A}^{\circ}$ 2016. This is an open-access article distributed under the terms of the Creative Commons Attribution License, which permits unrestricted use, distribution and reproduction in any medium, provided the original author and source are credited. 
demonstrated in Western populations, deserves to be validated by contribution to our own population [9]. It is with this objective that we are offered a prospective study in anesthesia department of H. Bourguiba hospital in Sfax, Tunisia.

\section{Methods}

This is a clinical study, prospective, consecutive, conducted after approval of the local ethics committee and informed consent of all patients. This study included 759 patients proposed for elective surgery and anesthetic consultation in $\mathrm{H}$. Bourguiba hospital in Sfax in collaboration with the hospital's pulmonology department Hedi Chaker on a 6-month period from 1 November 2011 to 1 May 2012 after informed consent.

The objective of this study was to validate the predictive value of "STOP BANG" questionnaire in screening for obstructive sleep apnea syndrome and identify patients at high risk of OSAS in consultation in a Tunisian hospital. The inclusion criteria for patients: age greater than 18 years; proposed for programmed urologic surgery, general surgery, cardiovascular and thoracic surgery, maxillofacial surgery, otorhinolaryngology surgery, ophthalmology and orthopedic. The exclusion criteria for patients: age less than 18 years; refusal to participate in the study; patient already known suffering from OSAS; severe chronic renal failure, stroke, dementia or Parkinson's syndrome; patients hospitalized for emergency surgery. Exclusion criteria of patients: non-compliance with the study protocol; uncooperative patients and patients lost.

Our study was conducted in two phases: a screening phase to the anesthesia consultation and the registration phase nocturnal ventilation during sleep in pulmonology department.

The first OSA screening phase by the questionnaire "STOP BANG" was held at the anesthesia consultation. During this phase, 759 patients included were informed of the study protocol. For each patient we collected the following data including: age, sex, weight $(\mathrm{kg})$, height $(\mathrm{cm})$ and body mass index (BMI), neck circumference $(\mathrm{cm})$, the main medical and surgical history and habits of patients, the criteria for intubation of patients, ASA classification of patients. In addition, each patient was asked to respond to an information document containing the STOP BANG translated questionnaire by the anesthetist. In this consultation, patients were informed of medical, social and professional consequences of OSAS, and their informed consent to participation in the study was taken. These patients were referred to the pulmonology department at H.Chaker hospital for ventilatory polygraphy whatever the estimated clinical probability was.

The second phase of the study took place at the pneumology service H.Chaker in Sfax. The patient is then presented in the service where it is equipped with a polygraph device recording his usual bedtime and spends the night in a single room dedicated exclusively to this type recording. The connection and disconnection of the device was made by the doctor.

Each polygraphy has been automatically analysed and must be followed by a manual screening conducted by the sleep specialist. The final interpretation report provides the following data: AHI (apnea hypopnea index); NDI (nocturnal desaturation index); the average duration of apnea and hypopnea; the average oxygen saturation; the minimum oxygen saturation; snoring and heart rate.

OSA is defined using the criteria of the AASM (American Academy of Sleep Medicine) by the presence of the criteria A or $\mathrm{B}$ and Criterion C: (A) Excessive daytime sleepiness not explained by other factors; (B) At least two of the following criteria is not explained by other factors: severe snoring and daily, feeling of suffocation or choking during sleep, non-restorative sleep, daytime fatigue, difficulty concentrating, nycturia (more than urination by night); (C) polysomnographic criterion or polygraph AHI $\geq 5 /$ hour of sleep. The severity of OSAS is based on two components: (1) The IAH: light if AHI between 5 and 15; moderate if AHI between 15 and 29 and if severe AHI greater than 30. (2) Daytime sleepiness (Appendix 1).

Statistical analysis was performed with the software: Statistical Package for Social Sciences (SPSS) for Windows (Version 20.0). Quantitative variables were expressed as mean \pm standard deviation or median depending on the nature of the distribution. Categorical variables were expressed as frequencies. For the comparison of averages, we used the Student's $t$ test or the $U$ Mann-Whitney test when the normality of distributions is not respected (non Gaussian distribution) or the size of one of the groups is low $(<10)$. Categorical variables were analysed by the Chi 2 test or the Fisher exact test whenever a workforce is low $(<5)$. Differences between results were considered significant for $\mathrm{P}<0.05$. For multiple comparisons we used the Bonferroni correction. The connection between two continuous variables was tested by Pearson correlation in case of Gaussian distribution and the Spearman correlation whenever the normality of distributions is not respected. To determine the various threshold values, we used the ROC (Receiver Operating Characteristic). The best threshold value is the value having the best sensitivity / specificity ratio. For the multivariate analysis we used binary logistic regression.

\section{Results}

The questionnaire was distributed to 759 consultants. Demographic characteristics, history and habits were summarized in Table 1. The screened population was composed of 150 patients divided according to clinical probability of OSAS estimated through the "STOP BANG" questionnaire in two groups (Figure 1): a group (1) consisting of 41 patients $(27.33 \%)$ in which the likelihood of OSAS is estimated low and group (2) consisting of 109 patients $(72.66 \%)$ in whom the clinical probability of OSAS is estimated high.

In our study using AHI which qualifies OSAS as light if it is between 5 and 15 events per hour, moderate if between 15 and 30 events per hour and severe if it is more than 30 events per hour: 23 patients $(15 \%)$ had a negative screening while 127 patients $(84.6 \%)$ had OSAS distributed as follows: 35 (27.5\%) Light, 43 $(33.8 \%)$ moderate and $49(38.5 \%)$ severe.

Using an AHI greater than 5 as a threshold for the diagnosis of OSAS, statistical analysis of the results showed that the majority of diagnosed OSAS were male with a clear predominance of 
Table 1. Characteristics of the Overall Study Population.

\begin{tabular}{|c|c|c|c|c|c|c|}
\hline & Total & Screened & $\mathrm{AHI}<5 / \mathrm{H}$ & $5 \leq \mathrm{AHI}<15 / \mathrm{H}$ & $15 \leq \mathrm{AHI}<30 / \mathrm{H}$ & $\mathrm{AHI} \geq 30 / \mathrm{H}$ \\
\hline Number & 759 & 150 & 23 & 35 & 43 & 49 \\
\hline $\operatorname{Sex}(M / F)$ & $453 / 306$ & $85 / 65$ & $8 / 15$ & $20 / 15$ & $27 / 16$ & $30 / 19$ \\
\hline Middle age (years) & $49(18-87)$ & $51(18-87)$ & $54.5(18-52)$ & $56.4(18-87)$ & $55.05(18-81)$ & $67(33-87)$ \\
\hline Middle BMI $\left(\mathrm{kg} / \mathrm{m}^{2}\right)$ & 29.47 & 32.09 & 29.80 & 32.10 & 32.95 & 34.75 \\
\hline $\mathrm{BMI} \geq 35\left(\mathrm{~kg} / \mathrm{m}^{2}\right)$ & 42 & 34 & 2 & 7 & 6 & 19 \\
\hline Hypertension & 204 & 64 & 7 & 13 & 15 & 29 \\
\hline Diabetes & 71 & 31 & 10 & 9 & 7 & 4 \\
\hline Tobacco & 162 & 54 & 4 & 11 & 10 & 29 \\
\hline
\end{tabular}

Figure 1. Distribution of Patients Diagnosed According to Clinical Probability of OSAS Through the Questionnaire "STOP BANG".

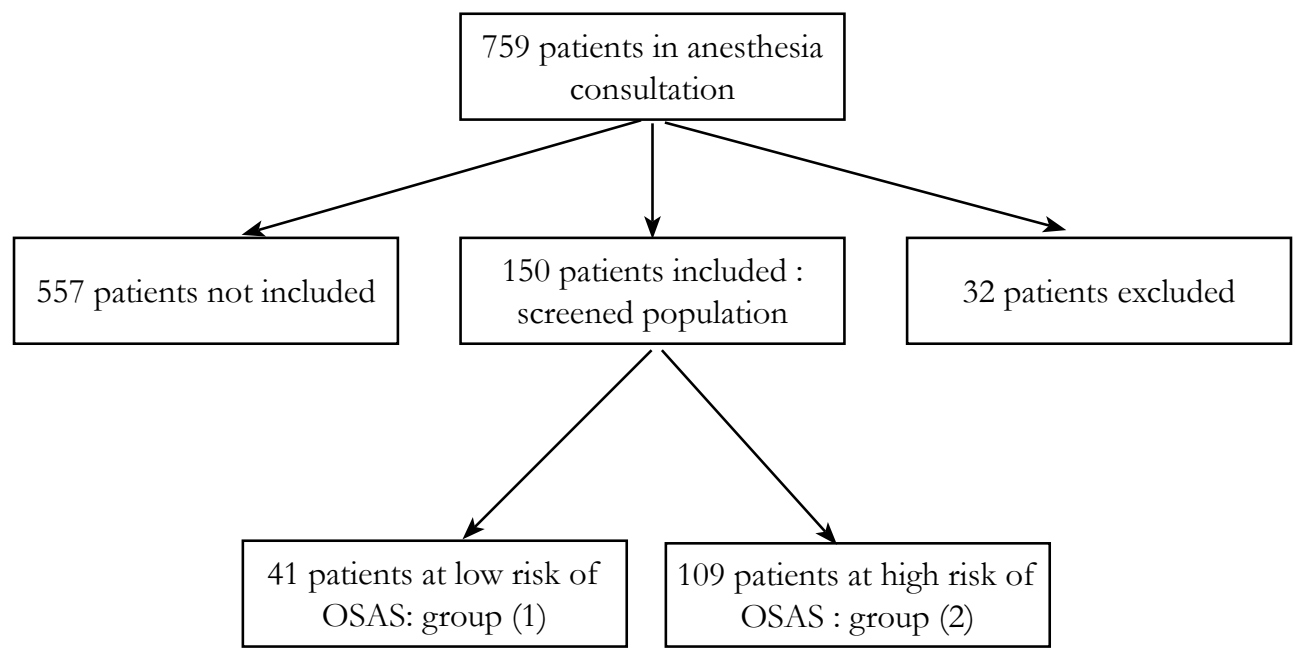

hypertensive patients.

Factors significantly associated with OSA are represented by age over 58 years, body mass index greater than $32 \mathrm{~kg} / \mathrm{m}^{2}$ and a neck circumference of greater than $40 \mathrm{~cm}$ (Table 2).

Statistical analysis of the screened population showed that the average score for men was 5.2 while that for women was 3.4. The maximum score for men was 8 against 7 for women. Snoring and observed apnea were reported more frequently by men, but the answers to drowsiness, BMI, age and systemic blood pressure were similar. The neck circumference was higher in men and AHI was more severe (Table 3). Taking into account the results of the polygraph records and according to the distribution of patients according to their score "STOP BANG", most severe OSAS were observed with the score 5 (14 cases).

Statistical analysis results showed that the odds of having OSA were higher when the STOP-Bang score increased. This trend was not the same in different stages of OSAS. The likelihood of having severe OSA continues to increase with increasing score of the questionnaire "STOP BANG", while for the other stages it decreased.

The results showed that the sensitivity of the score STOP BANG questionnaire of the order of $87.1 \%$, the specificity was $47.8 \%$, with positive and negative predictive values $90 \%$ and $40.74 \%$ respectively. The sensitivity and the score of the VPN increased significantly with increasing AHI, are respectively $91.3 \%$ and $70.37 \%$ for $\mathrm{AHI} \geq 15 / \mathrm{H}, 93.9 \%$ and $88.9 \%$ for $\mathrm{AHI} \geq 30 / \mathrm{M}$ (Table 4).

Statistical analysis of the results showed that the sensitivity, specificity, PPV and NPV vary by IAH and score STOP BANG questionnaire. For one score increase to 8 sensitivity decreased while specificity increased. This trend was the same in the different stages of OSAS (Table 5).

At the end of the study 49 patients of the population screened with a positive result of the polygraph recording; 38.5\% were paired before surgery. While the surgical treatment in otorhinolaryngology department before the procedure was proposed for 43 patients $(33.85 \%)$. The lifestyle changes were suggested for all OSAS severity degrees.

\section{Discussion}

The validity of the questionnaire "STOP BANG" in the Western population prompted to seek the same results in our population.

OSA screening is primarily based on clinical suspicion often associated with predisposing factors. These elements have been included in several screening questionnaires. Of these, the questionnaire "STOP BANG" used in our study [10, 8]. For a surgical population, ease of use is very important for a screening tool. "STOP BANG" questionnaire is simple and easy to use 
Table 2. Patient characteristics according to AHI.

\begin{tabular}{|c|c|c|c|}
\hline & AHI < 5/H & AHI $\geq \mathbf{5 / H}$ & $\mathbf{P}$ \\
\hline Sex $(\mathrm{M} / \mathrm{F})$ & $8 / 15$ & $77 / 50$ & 0.002 \\
\hline Age (years) & $47 \pm 12$ & $55 \pm 14$ & 0.04 \\
\hline BMI $\left(\mathrm{kh} / \mathrm{m}^{2}\right)$ & $29 \pm 6$ & $33 \pm 6$ & 0.037 \\
\hline Hypertension $(\mathrm{n})$ & 7 & 57 & 0.0001 \\
\hline Neck circumference $(\mathrm{cm})$ & $36 \pm 3$ & $40 \pm 5$ & 0.023 \\
\hline Minimal Sp $\mathrm{O}_{2}(\%)$ & $88 \pm 8$ & $81 \pm 10$ & 0.001 \\
\hline
\end{tabular}

Table 3. Descriptive Statistics of the Population According to the Questionnaire "STOP BANG" and AHI.

\begin{tabular}{|c|c|c|c|}
\hline & Total $(\mathbf{n}=\mathbf{1 5 0})$ & Male $(\mathbf{n}=\mathbf{8 5})$ & Female (n=65) \\
\hline Snoring $(\mathrm{n})$ & 93 & 59 & 33 \\
\hline Dowsiness $(\mathrm{n})$ & 133 & 74 & 59 \\
\hline Observed respiratory pauses $(\mathrm{n})$ & 72 & 51 & 21 \\
\hline Hypertension $(\mathrm{n})$ & 63 & 35 & 27 \\
\hline BMI $\left(\mathrm{kg} / \mathrm{m}^{2}\right)$ & 32.09 & 33.4 & 35 \\
\hline Age $($ years $)$ & 51 & 50.1 & 52 \\
\hline Neck circumference $(\mathrm{cm})$ & $39 \pm 6$ & $42 \pm 8$ & $38 \pm 4$ \\
\hline Sex $(\%)$ & 100 & 56.6 & 43.4 \\
\hline STOP BANG score & 4.3 & 5.2 & 3.4 \\
\hline AHI $(/ \mathrm{H})$ & $33 \pm 30$ & $38 \pm 30$ & $26.5 \pm 28$ \\
\hline
\end{tabular}

Table 4. Sensitivity, Specificity, PPV and NPV of the Questionnaire "STOP BANG" According to AHI.

\begin{tabular}{|c|c|}
\hline IAH & Values (\%) \\
\hline \multicolumn{2}{|c|}{ AHI > $\mathbf{5 / H}$} \\
\hline Sensibility & 87.1 \\
Specificity & 47.8 \\
PPV & 90 \\
NPV & 40.74 \\
\hline \multicolumn{2}{|c|}{ AHI > 15/H } \\
\hline Sensibility & 91.3 \\
Specificity & 32.7 \\
PPV & 68.3 \\
NPV & 70.37 \\
\hline \multicolumn{2}{|c|}{ AHI > 30/H } \\
\hline Sensibility & 93.9 \\
Specificity & 24 \\
PPV & 37.7 \\
NPV & 88.9 \\
\hline
\end{tabular}

features eight questions, filled at least 2 minutes, which makes this form particularly suitable for anesthesia consultation [8].

Polysomnography considered the "gold standard" for diagnosis of OSAS is an expensive examination, complex and requires significant time for recording [11]. Ventilatory polygraphy has shown its reliability for the positive diagnosis of OSAS with less cost, shorter time and easier use [12]. In our study, we used the ventilator polygraphy for the diagnosis of OSAS.

In most previous studies, polygraphy recordings were used to validate the questionnaires. However, the collection of data was generally not blinded to the score of the questionnaire [13-16]. In our study, polygraphy in pulmonology department was used to evaluate our questionnaire. The staff of this review was blinded score of the questionnaire "STOP BANG". This practice avoids the bias rating of the polygraph. Chung et al., have shown, in a population of 746 patients, sensitivity of the questionnaire which was around $93 \%$ for detection of OSAS and 100\% for detecting moderate and severe OSA [17]. The questionnaire "STOP BANG" has excellent sensitivity when at least three criteria are positive: $84 \%$ sensitivity for detecting OSA with an AHI greater than 5 and $93 \%$ for OSAS with an AHI greater than 15 . However, this questionnaire has poor specificity (50\%) [8]. In our study the sensitivity of the score STOP BANG questionnaire was to the order of $87.1 \%$, specificity was $47.8 \%$, with positive and negative 
Table 5. Sensitivity, Specificity, PPV and NPV According to AHI and the Score of the Questionnaire "STOP BANG".

\begin{tabular}{|c|c|c|c|c|}
\hline & Sensibility (\%) & Specificity (\%) & PPV (\%) & NPV (\%) \\
\hline \multicolumn{5}{|c|}{$\mathrm{AHI} \geq 5 / \mathrm{H}$} \\
\hline 1 & 83 & 40 & 62 & 66 \\
\hline 2 & 62 & 45 & 62.5 & 45 \\
\hline 3 & 54 & 73 & 82 & 40 \\
\hline 4 & 44 & 83 & 90 & 30 \\
\hline 5 & 32.2 & 90 & 93.7 & 22.12 \\
\hline 6 & 16.22 & 90.9 & 90 & 17.7 \\
\hline 7 & 9.7 & 95.6 & 92 & 16.5 \\
\hline 8 & 3.5 & 100 & 100 & 15.7 \\
\hline \multicolumn{5}{|c|}{$\mathrm{AHI} \geq 15 / \mathrm{H}$} \\
\hline 1 & 100 & 37.7 & 37.5 & 100 \\
\hline 2 & 62.5 & 42 & 31.2 & 72.7 \\
\hline 3 & 60 & 63 & 52 & 70.3 \\
\hline 4 & 50 & 73 & 64.5 & 60 \\
\hline 5 & 39.4 & 83.6 & 76.5 & 50.6 \\
\hline 6 & 20 & 90.7 & 75 & 43.36 \\
\hline 7 & 11.2 & 94.7 & 76.9 & 40.6 \\
\hline 8 & 3.26 & 98.3 & 75 & 39.04 \\
\hline \multicolumn{5}{|c|}{$\mathrm{AHI} \geq 30 / \mathrm{H}$} \\
\hline 1 & 100 & 30 & 12.5 & 100 \\
\hline 2 & 66.6 & 41.6 & 12.5 & 90.9 \\
\hline 3 & 57.1 & 55.8 & 17.4 & 88.9 \\
\hline 4 & 56 & 66.1 & 29 & 86 \\
\hline 5 & 48 & 79.27 & 46.8 & 80.25 \\
\hline 6 & 24.3 & 89.13 & 50 & 72.57 \\
\hline 7 & 12.7 & 92.9 & 46.15 & 69.17 \\
\hline 8 & 4 & 98.02 & 50 & 67.8 \\
\hline
\end{tabular}

predictive value (NPV) $90 \%$ and $40.74 \%$ respectively. The sensitivity and the score of the NPV increased significantly with increasing AHI, are respectively $91.3 \%$ and $70.37 \%$ for $\mathrm{AHI} \geq 15$ / $\mathrm{H}, 93.9 \%$ and $88.9 \%$ for $\mathrm{AHI} \geq 30 / \mathrm{H}$. While the specificity decreased significantly with increasing $\mathrm{AHI}$, it is $32.7 \%$ for $\mathrm{AHI}$ $\geq 15 / \mathrm{M}$ and $24 \%$ for $\mathrm{AHI} \geq 30 / \mathrm{H}$.

Recent studies have shown that, along with the increase of the score "STOP BANG", there was a corresponding increase in the likelihood of having OSA and this for different stages [17]. "STOP BANG" questionnaire was validated in two studies in patients referred to sleep centers [18, 19]. Farney showed that this questionnaire can be used to estimate the probabilities of the various stages of OSAS, mostly in severe cases [18]. In our study the probabilities of having a OSAS were higher when the score of the questionnaire "STOP BANG" has increased. This trend was not the same in different stages of OSAS. With a score $\geq$ 4 , the probability of having severe OSAS increases continuously, whereas for the other stages it decreased. In the same study, the probability of severe OSA was $84 \%$ with a score of 8 [18]. In our study results also showed similar associations between the odds of having severe stages and score "STOP BANG". For a score of 8 , the probability was $74 \%$. This lower value may be due to the difference in the population studied, our patients were preoperative patients. Patients in the study of Faney were referred to sleep centers that have a high prevalence of severe OSA. A score of "STOP BANG" greater than or equal to 3 showed a high sensitivity and good NPV to detect OSAS to moderate and severe stages, this threshold may be useful in a surgical population with a high prevalence of severe OSAS such as bariatric surgery patients [17]. This eliminates the possibility of severe or moderate OSA with a score of "STOP BANG" 0 to 2 . Our results were consistent with the literature. With a score $\geq 3$ the sensitivity and NPV were respectively in the moderate stages of $91.3 \%$ and $70.37 \%$ for severe stages they were $93.9 \%$ and $88.9 \%$. On the other hand, the scores of 5 to 8 have a high specificity to detect moderate and severe OSAS [17]. In our study the specificity increases with increasing the score with high specificity for scores of 5 to 8 and this for different stages of OSAS.

STOP BANG questionnaire was presented as a preoperative evaluation model [8]. The results of this study validate advantage of the value of this questionnaire as a screening tool in surgical patients of North African population.

\section{Conclusion}

In our study, the results showed that the sensitivity of the questionnaire was approximately $87.1 \%$, specificity was $47.8 \%$, with positive and negative predictive values $90 \%$ and $40.74 \%$ respectively. The score of the sensitivity increased significantly with increasing AHI, it was $91.3 \%$ for $\mathrm{AHI} \geq 15 / \mathrm{H}$ and $93.9 \%$ for $\mathrm{AHI} \geq 30$ / M. Similarly, the score of the VPN has increased in parallel with the increase in AHI, it was $70.37 \%$ for AHI $\geq 15$ / $\mathrm{M}$ and $88.9 \%$ for $\mathrm{AHI} \geq 30 / \mathrm{H}$. The sensitivity, specificity, PPV and NPV vary according to the AHI and the score of the questionnaire "STOP BANG". This study supports the validity of 
the "STOP BANG" questionnaire and requires a larger number of patients to better validate this questionnaire in our population.

\section{References}

[1]. AASM (1999) Sleep-related breathing disorders in adults: recommendations for syndrome definition and measurement techniques in clinical research. The Report of an American Academy of Sleep Medicine Task Force. Sleep. 22(5): 667-689.

[2]. Escourrou P, Roisman GL (2010) Épidémiologie du syndrome d'apnéeshypopnées obstructives du sommeil de l'adulte et de ses complications. Médecine du Sommeil. 7(4): 119-128. doi:10.1016/j.msom.2010.09.002.

[3]. Punjabi NM, Caffo BS, Goodwin JL, Gottlieb DJ, Newman AB, et al., (2009) Sleep-disordered breathing and mortality: a prospective cohort study. PLoS Med. 6(8):e1000132. doi:10.1371/journal.pmed.1000132.

[4]. Vijayan VK (2012) Morbidities associated with obstructive sleep apnea. Expert Rev Respir Med. 6(5): 557-566. doi:10.1586/ers.12.44.

[5]. Peterson GN, Domino KB, Caplan RA, Posner KL, Lee LA, et al., (2005) Management of the difficult airway: a closed claims analysis. Anesthesiology. 103(1): 33-39.

[6]. Kurtipek O, Isik B, Arslan M, Unal Y, Kizil Y, et al., (2012) A study to investigate the relationship between difficult intubation and prediction criterion of difficult intubation in patients with obstructive sleep apnea syndrome. J Res Med Sci. 17(7): 615-620.

[7]. Semelka M, Wilson J, Floyd R (2016) Diagnosis and Treatment of Obstructive Sleep Apnea in Adults. Am Fam Physician. 94(5): 355-360.

[8]. Chung F, Yegneswaran B, Liao P, Chung SA, Vairavanathan S, et al., (2008) STOP questionnaire: a tool to screen patients for obstructive sleep apnea. Anesthesiology. 108(5): 812-821. doi:10.1097/ALN.0b013e31816d83e4.

[9]. Chung F, Abdullah HR, Liao P (2016) STOP-Bang Questionnaire: A Practi- cal Approach to Screen for Obstructive Sleep Apnea. Chest. 2016;149(3): 631-638. doi:10.1378/chest.15-0903.

[10]. Ramachandran SK, Josephs LA (2009) A meta-analysis of clinical screening tests for obstructive sleep apnea. Anesthesiology. 110(4): 928-939. doi:10.1097/ALN.0b013e31819c47b6.

[11]. Pack AI (2004) Sleep-disordered breathing: access is the issue. Am J Respir Crit Care Med. 169(6): 666-667. doi:10.1164/rccm.2401008.

[12]. Thurnheer R, Bloch KE, Laube I, Gugger M, Heitz M, et al., (2007) Respiratory polygraphy in sleep apnoea diagnosis. Report of the Swiss respiratory polygraphy registry and systematic review of the literature. Swiss Med Wkly. 137(5-6): 97-102. doi:2007/05/smw-11654.

[13]. Rowley JA, Aboussouan LS, Badr MS (2000) The use of clinical prediction formulas in the evaluation of obstructive sleep apnea. Sleep. 23(7): 929-938.

[14]. Roth T, Zammit G, Kushida C, Doghramji K, Mathias SD, et al., (2002) A new questionnaire to detect sleep disorders. Sleep Med. 3(2): 99-108.

[15]. Maislin G, Pack AI, Kribbs NB, Smith PL, Schwartz AR, et al., (1995) A survey screen for prediction of apnea. Sleep. 18(3): 158-166.

[16]. Kapuniai LE, Andrew DJ, Crowell DH, Pearce JW (1988) Identifying sleep apnea from self-reports. Sleep. 11(5): 430-436.

[17]. Chung F, Subramanyam R, Liao P, Sasaki E, Shapiro C, Sun Y (2012) High STOP-Bang score indicates a high probability of obstructive sleep apnoea. Br J Anaesth. 108(5):768-775.doi:10.1093/bja/aes022.

[18]. Farney RJ, Walker BS, Farney RM, Snow GL, Walker JM (2011) The STOPBang equivalent model and prediction of severity of obstructive sleep apnea: relation to polysomnographic measurements of the apnea/hypopnea index. J Clin Sleep Med. 7(5): 459-465B. doi:10.5664/JCSM.1306.

[19]. Silva GE, Vana KD, Goodwin JL, Sherrill DL, Quan SF (2011) Identification of patients with sleep disordered breathing: comparing the four-variable screening tool, STOP, STOP-Bang, and Epworth Sleepiness Scales. J Clin Sleep Med. 7(5): 467-472. doi:10.5664/JCSM.1308. 